www.jmscr.igmpublication.org

Index Copernicus Value: 79.54

ISSN (e)-2347-176x ISSN (p) 2455-0450

crossrefDOI: https://dx.doi.org/10.18535/jmscr/v7i2.167

\title{
Berardinelli’s Syndrome- Anaesthetic Considerations
}

\author{
Authors \\ Dr Gadre Vaijayanti Nitin 1 , Dr Badole Usha Ratan ${ }^{2}$ \\ ${ }^{1}$ Associate Professor, Grant GMC, Mumbai, Maharashtra, India \\ 303-C Aarav, Opp Y.C. Chavan Natyagriha. Behind Mhatoba Mandir, Kothrud, PUNE 411038 India \\ Email: vaijayantigadre@hotmail.com \\ ${ }^{2}$ Professor and Head, Grant GMC, Mumbai, Maharashtra, India \\ Email: vaijayantigadre@hotmail.com
}

\begin{abstract}
Berardinelli's Syndrome or Laurence Seip Syndrome is a rare inherited disorder presenting in early childhood. Glucose and fat metabolism are mainly deranged with clinical presentation of generalized lipoatrophy, hypertriglyceridemia and insulin resistance in early childhood. There is no curative treatment, anticipation and symptomatic treatment of complications is the reported management. Precise metabolic control was challenging during anesthesia for a seven year old child with multisystem involvement like an aged patient.
\end{abstract}

Keywords: Berardinelli's Syndrome, Laurence Seip Syndrome, inherited metabolic disorder.

\section{Introduction}

Berardinelli's Syndrome is a rare inherited autosomal recessive disorder. Presenting in early childhood, there is lack of adipose tissue with characteristic clinical picture of hypertriglyceridemia, hyperglycemia, and insulin resistance with microvascular complications of diabetes mellitus. Carbohydrate and lipid metabolism are affected resulting in attention deficit, learning disorders, hypertrophic cardiomyopathy, acanthosis nigricans, acromegaly and precocious puberty. The extent of fat loss determines the severity of metabolic complications.

\section{Case Report}

$118 \mathrm{~cm}$ tall, $21 \mathrm{~kg}, 7$ years old male child presented with throat pain, aggravated during cold and relieved after medication. Throat pain was not associated with fever, nasal bleed, dysphagia or earache. Mother told about progressive skin darkening at the nape of neck, axilla and groin. Patient was the second child of a nonconsanguineous marriage, delivered at home. Mother had negative history of allergy, addictions or infection in antenatal period. Neonatal period, infancy and early childhood were uneventful with timely immunization. Motor milestones were normal but scholastic performance was poor. Child had old man like face, 20 permanent teeth, flat cheeks and appearance of moustache. Axilla and nape of neck showed dark pigmentation. Body was muscular, hands and feet were large with enlarged bony prominences (Figure-1,2). 


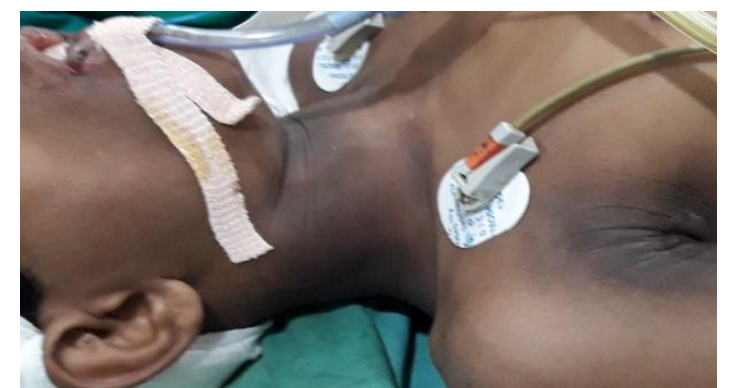

Fig.1 Shows dark pigmentation of neck and axilla

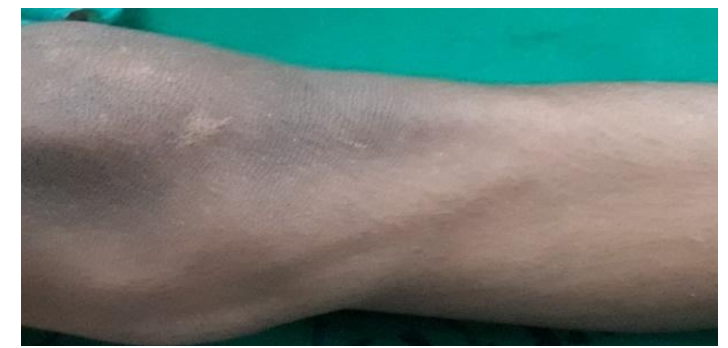

Fig.2 Shows enlarged bony prominence

Abdomen examination showed non-tender liver enlargement. ENT examination revealed grade 3 bilateral tonsillitis. Patient was admitted to diagnose syndromic state and treat tonsillitis. Haemoglobin $(15.9 \mathrm{gm} \%)$, serum levels of creatinine $(0.8 \mathrm{mg} \%)$, urea $(19 \mathrm{mg} \%)$, bilirubin $(0.2 \mathrm{mg} \%)$ and insulin fasting $(8.59 \mu \mathrm{IU} / \mathrm{ml}$, normal-2 to 2.5$)$ were normal. Values that were deranged were serum insulin postprandial $(305 \mu \mathrm{IU} / \mathrm{ml}$, normal- 22 to 75 after two hours), $\mathrm{HbA}_{1} \mathrm{C}-6.5$, serum hormones like $\mathrm{FSH}$ $=0.53(2.5-10 \mathrm{~m} \mathrm{IU} / \mathrm{ml}), \mathrm{LH}=0.05(2.5-10 \mathrm{~m}$ $\mathrm{IU} / \mathrm{ml})$ and Total T3= $161.10(0.4-24.1 \mathrm{ng} / \mathrm{dl})$. Total $\mathrm{T} 4=9.21(0.4-13.3 \mathrm{ng} / \mathrm{dl})$ and $\mathrm{TSH}=1.304(0.45-$ $4.5 \mu \mathrm{IU} / \mathrm{ml})$ were normal. Patient was put on tablet metformin 500mg once daily for a week and advised to continue half tablet twice daily in future. He had hypertriglyceridemia $(159 \mathrm{mg} / \mathrm{dl})$; serum cholesterol: HDL ratio $=4.95$ (normal is $<5$ ), serum triglyceride: $\mathrm{HDL}$ ratio $=5.78$ (desirable is $<3$ ), SGOT= 59 (8-40U/liter), SGPT=140 (5-35 U/liter). Thus patient had poor control of diabetes along with liver dysfunction. Serum CPK-MB levels were high (148 U/liter normal= 31-150) indicating highly active skeletal muscles. Raised T3 and normal T4 and TSH show T3 toxicosis with non-thyroidal illness.

Patient received general anaesthesia for tonsillectomy with propofol 50mg, atracurium $0.5 \mathrm{mg} / \mathrm{kg}$; intubated with cuffed $5.5 \mathrm{~mm}$ tube under direct vision laryngoscopy after premedication with intravenous glycopyrrolate $0.1 \mathrm{mg}$, ondansetron $4 \mathrm{mg}$, midazolam $0.5 \mathrm{mg}$ and fentanyl $50 \mu \mathrm{g}$. Sevoflurane $2 \%$ in $\mathrm{O}_{2}: \mathrm{N}_{2} \mathrm{O}, 50: 50$ were used for maintenance. Patient was reversed and extubated after thorough suction, removal of throat pack and return of active reflexes.

\section{Discussion}

Laurence-Seip syndrome is alternatively termed as Berardinelli- Seip Congenital Lipodystrophy type 2. Loss of function of gene encoding Seipin results in change in pattern of lipid droplets in cells with decrease in their size and number. ${ }^{[1]}$

Seipin is a protein in endoplasmic reticulum localized near lipid droplets; one loop is in the lumen of endoplasmic reticulum, two transmembrane and two terminals in the cytoplasm. It helps lipid droplet maintenance in lipid catabolism and prevents ectopic lipid droplet formation in non-adipose tissue. Content of Seipin is high in adipose tissue and low in liver and rarely detectable in muscles. It regulates central energy homeostasis and is found highly expressed in cortical and spinal cord motor neurones. Over expression of mutated Seipins activate autophagy and form large vacuoles in cytoplasm. Deficiency causes lipodystrophy as Seipin acts as a scaffolding protein to bring together interacting partners of lipid metabolism during lipid droplet formation and adipocyte development. ${ }^{[2]}$

Our patient had generalized absence of subcutaneous fat giving old man like appearance in a seven year old. There was associated hypertriglyceridemia, poor glycemic control and high CPK-MB levels posing risk of cirrhosis, pancreatitis and coronary artery disease at young age.

According to Zhou et al biochemical assays revealed increased lipolysis and fatty acid oxidation in adipose tissues; there is browning of white adipose tissue owing to induction of cAMP signaling upon $\mathrm{Bscl}_{2}$ deletion (Berardinelli Seip Congenital Lipodystrophy type 2). This causes reduced food intake, down regulated beta 3 
adrenergic adipose receptors, upregulation of browning induced energy expenditure and thermogenesis. ${ }^{[3]}$

Hormone leptin secreted by adipocytes regulates food intake and energy expenditure. Peterson et al performed body fat composition study and muscle biopsy; liquid chromatography tandem mass spectrometry was performed to assess long chain fatty acyl CoA from biopsy samples. Authors found almost complete lack of subcutaneous and visceral fat in subjects under study with significant intrahepatic and intravascular triglyceride lipolysis. Increased insulin resistance with high intracellular content of fatty acids was attributed to fatty acyl CoA mediated activation of protein kinase causing phosphorylation of insulin receptors. This activated glucose translocation, increased hepatic gluconeogenesis resulted in hepatic steatosis. Leptin treatment improved glycemic control and increased insulin responsiveness inferred from $85 \%$ decrease in hepatic triglyceride and $30 \%$ fall in intramyocellular triglyceride content. Leptin improved insulin stimulated peripheral glucose uptake which reduced hepatic and muscle triglyceride content after treatment. They reported recombinant leptin therapy useful for improved glycemic control. Correction of metabolic imbalance allowed large reduction in anti-diabetic therapy. ${ }^{[4]}$

Hyperinsulinemia causes increased growth velocity, advanced bone age, muscular hypertrophy and masculine body built; as were also the findings in our patient. Blood sugar levels were controlled with pre-operative metformin. Difficult intubation was expected due to tonsillar hypertrophy. Propofol induction and maintenance with sevoflurane was done anticipating the risk of delayed awakening in hypertriglyceridemia. ${ }^{[5],[6]}$

Ferraria $^{[7]}$ et al suggested symptomatic control of complications to slow down life-threatening progression of the disease. They reported the follow up of cases presented at infancy with hypertension and cardiac hypertrophy, developed hepatomegaly, acromegaly and acanthosis nigricans at 5 years followed by lipoatrophy and muscle hypertrophy by 11 years. Low leptin levels were demonstrated; defective glucose metabolism affected storage of glucose as fats causing excessive glycogen storage in skeletal and cardiac muscles and in liver. Control of insulin resistance with metformin reduced appetite and controlled hepatic steatosis. Hypolipidemic diet, diuretics for nephron-protection and fenofibrate for hypertriglyceridemia were useful.

\section{Conclusion}

Systemic involvement causing coronary and renal artery disease and diabetes need special attention until adulthood. Early diagnosis of precocious puberty prevents emotional problems of a growing child. Early institution of anti-diabetic drug therapy helped metabolic control peri-operatively in this unusual case.

\section{References}

1. Emilie Boutet, Haquima EI Mourabit, Matthieu Prot, Mona Nemani et al. "Seipin Deficiency alters Fatty Acid De-saturation and Lipid Droplet Formation in BerardinelliSeip Congenital Lipodystrophy." Biochemie; 91(6): 796-803, 2009.

2. Kenneth Wee, Wulin Yang, Shigeki Sugii, Weiping Han. "Towards Mechanistic Understanding of Lipodystrophy and Seipin Functions.” Bioscience Reports; 34(5): 583591, 2014.

3. Hongyi Zhou, Xinnuo Lei, Tyler Benson, James Mintz et al. "Berardinelli Seip Congenital Lipodystrophy type 2 regulated adipocytolipolysis, browning and energy balance in adult animals." Journal of Lipid Research; 56: 1912-1925, 2015.

4. Peterson K, Oral E, Dofour S. "Leptin Reverses Insulin Resistance and Hepatic Steatosis in Patients with Severe Lipodystrophy." Journal Clinical Investigation; 109: 1345-50, 2002.

5. Steen ER, De Baerdemaeker LEC, Van Limmen E, Wouters P. "Some Pharmacokinetics of Sevoflurane in a Child 
with Severe Lipodystrophy." A \& A Case Reports; 2: 61-64, 2014.

6. Bennett T, Allford M. "Delayed Emergence from Anaesthesia in a Child with Congenital Generalized Lipodystrophy (BerardinelliSeip Syndrome)." Paediatric Anaesth; 22: 299-300, 2012.

7. Nelia Ferraria, Cristina Pedrosa, Daniela Amaral and Lurdes Lopes. "BerardinelliSeip syndrome: Highlight of Treatment Challenge." British Medical Journal Case Reports doi: 10.1136/ber-2012-007734, 2013. 